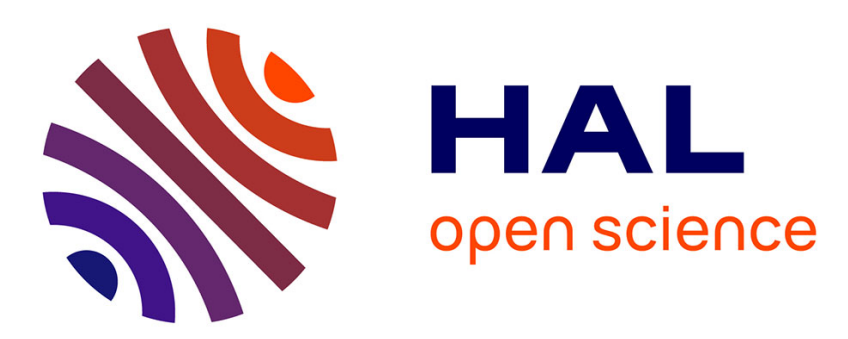

\title{
Observer Design for a Coupled ODE-PDE System from a Wellbore Reservoir Drilling Model
}

Leobardo Camacho-Solorio, Naveen Velmurugan, Florent Di Meglio, Miroslav

Krstic

\section{- To cite this version:}

Leobardo Camacho-Solorio, Naveen Velmurugan, Florent Di Meglio, Miroslav Krstic. Observer Design for a Coupled ODE-PDE System from a Wellbore Reservoir Drilling Model. 58th Conference on Decision and Control CDC 2019, Dec 2019, Nice, France. hal-02437365

\section{HAL Id: hal-02437365}

https://hal-mines-paristech.archives-ouvertes.fr/hal-02437365

Submitted on 4 Mar 2020

HAL is a multi-disciplinary open access archive for the deposit and dissemination of scientific research documents, whether they are published or not. The documents may come from teaching and research institutions in France or abroad, or from public or private research centers.
L'archive ouverte pluridisciplinaire HAL, est destinée au dépôt et à la diffusion de documents scientifiques de niveau recherche, publiés ou non, émanant des établissements d'enseignement et de recherche français ou étrangers, des laboratoires publics ou privés. 


\title{
Observer Design for a Coupled ODE-PDE System from a Wellbore Reservoir Drilling Model
}

\author{
Leobardo Camacho-Solorio, Naveen Velmurugan, Florent Di Meglio and Miroslav Krstic
}

\begin{abstract}
The problem of state estimation for a coupled ODE-PDE system is addressed here by means of the backstepping method for PDEs. The ODE is a finite-dimensional, linear, and time-invariant system and the PDE is a linear radial diffusion equation with Neumann and Robin boundary conditions. The coupling appears at one of the boundaries of the PDE and is bidirectional. More precisely, the ODE state appears in one of the boundary conditions of the PDE and the value of the PDE state at the boundary is an input to the ODE. Measurements of the ODE output are available, while the state of the PDE is out of sight. The estimate is defined as the state of an observer; constructed as a copy of the coupled system ODEPDE with output error feedback. This study is motivated by the influx estimation problem from a wellbore-reservoir model used in managed pressured drilling applications.
\end{abstract}

\section{INTRODUCTION}

The problem of state estimation for a coupled ODE-PDE system is addressed here by means of the backstepping method for PDEs [1]. The ODE is a finite dimensional, linear, and time-invariant system and the PDE is a linear radial diffusion equation with Neumann and Robin boundary conditions. The coupling appears at one of the boundaries of the PDE and is bidirectional. More precisely, the ODE state appears in one of the boundary conditions of the PDE and the value of the PDE state at the boundary is an input to the ODE. Measurements of the ODE output are available, while the state of the PDE is out-of-sight. The estimate is defined as the state of an observer; constructed as a copy of the coupled system ODE-PDE with output error injection. The convergence of the estimate follows the stability properties of the estimation error system. Observer gains are selected specifically to guarantee the exponential stability of the estimation error system. To guarantee the existence and the invertibility of the backstepping transformation the wellposedness of a cascade hyperbolic PDE-DAE system is derived.

The purpose of this study is to provide a solution to the state estimation problem for a wellbore-reservoir model used in managed pressured drilling (MPD) operations [2]. An ODE is used to described pressure dynamics of a fluid along the wellbore and a radial diffusion equation is used to described the diffusion of the fluid in a porous reservoir.

L. Camacho-Solorio and M. Krstic are with the Department of Mechanical and Aerospace Engineering, University of California San Diego, La Jolla, CA 92093-0411, USA. Icamacho@ucsd.edu, krsticeucsd.edu

N. Velmurugan and F. Di Meglio are with the Centre Automatique et Systèmes, MINES ParisTech, PSL Research University, 60 bd St-Michel, 75272 Paris Cedex 06 naveen.velmurugan@mines-paristech.fr, florent.di_meglio@mines-paristech.fr
The bidirectional coupling between the ODE and the PDE arises from conservation laws and the continuity of physical quantities in the model.

Control and estimation problems for cascaded PDE-ODE systems including transport, heat, and wave PDEs were studied in [3], [4, Part IV], [5], [6], and [9]. An observer for cascaded hyperbolic PDE-ODE system was derived in [12], to estimate flow, pressure and down hole rate of circulation loss in oil well drilling application. A cascaded stabilization cascaded ODE-Schroedinger equation was studied in [11]. State and output feedback for a coupled diffusion ODE system was developed in [10] and state and output feedback for sandwiched ODE-PDE-ODE system was develop in [8].

\section{PROBLEM STATEMENT}

In this section, we construct an observer and derive observability conditions for a coupled ODE-PDE system. The ODE is a linear time invariant $n$-dimensional system

$$
\begin{aligned}
\frac{d x}{d t}(t) & =A x(t)+B u(a, t), \\
y(t) & =C x(t),
\end{aligned}
$$

with $A \in \mathbb{R}^{n \times n}, B \in \mathbb{R}^{n \times 1}, C \in \mathbb{R}^{1 \times n}$, for $t \in(0, T]$ and initial conditions $x_{0} \in \mathbb{R}^{n}$. The PDE is a radial-diffusion equation

$$
\partial_{t} u(r, t)=\frac{\epsilon}{r^{m-1}} \partial_{r}\left(r^{m-1} \partial_{r} u(r, t)\right),
$$

for $r \in(a, b), t \in(0, T]$, with diffusion coefficient $\epsilon>0$ and some integer parameter $m>0$, related to the geometry of the underling physical problem. Boundary conditions are

$$
\begin{aligned}
& \partial_{r} u(a, t)=\beta u(a, t)+D x(t), \\
& \partial_{r} u(b, t)=0,
\end{aligned}
$$

with $\beta>0$, and initial conditions $u_{0} \in C(a, b)$. The system is understood as a dynamic system with combined state $x \in C\left([0, T] ; \mathbb{R}^{n}\right), u \in C\left([0, T] ; \mathcal{L}^{2}(a, b)\right)$, and output $y \in C([0, T] ; \mathbb{R})$. The estimation objective is to compute an estimate $\widehat{x}, \widehat{u}$ from measurements $y(t) \in \mathbb{R}$ with exponential convergence in the sense of a norm. The proposed observer is a copy of (1)-(5) with output error feedback, that is

$$
\begin{aligned}
\frac{d \widehat{x}}{d t}(t) & =A \widehat{x}(t)+B \widehat{u}(a, t)+L(\widehat{y}(t)-y(t)), \\
\widehat{y}(t) & =C \widehat{x}(t),
\end{aligned}
$$

for $t \in(0, T]$, with observer gain $L=\left[l_{1}, l_{2}, \cdots, l_{n}\right]$, initial conditions $\widehat{x}_{0} \in \mathbb{R}^{n}$, and

$\partial_{t} \widehat{u}(r, t)=\frac{\epsilon}{r^{m-1}} \partial_{r}\left(r^{m-1} \partial_{r} \widehat{u}(r, t)\right)+l_{n+1}(r)(\widehat{y}(t)-y(t))$, 
for $r \in(a, b), t \in(0, T]$, boundary conditions

$$
\begin{aligned}
& \partial_{r} \widehat{u}(a, t)=\beta \widehat{u}(a, t)+D \widehat{x}(t)+l_{n+2}(y(t)-\widehat{y}(t)), \\
& \partial_{r} \widehat{u}(b, t)=l_{n+3}(y(t)-\widehat{y}(t)),
\end{aligned}
$$

with observer gains $l_{n+1} \in \mathcal{L}^{2}(a, b), l_{n+2} \in \mathbb{R}, l_{n+3} \in \mathbb{R}$, and initial conditions $\widehat{u}_{0} \in \mathcal{L}^{2}(0,1)$. The estimation error is defined as the difference between the state $x, u$ and the observer state $\widehat{x}, \widehat{u}$, that is

$$
\begin{aligned}
\widetilde{x}(t) & =x(t)-\widehat{x}(t), \\
\widetilde{u}(r, t) & =u(r, t)-\widehat{u}(r, t) .
\end{aligned}
$$

The estimation error $\widetilde{x}, \widetilde{u}$ is a solution of the estimation error system

$$
\begin{aligned}
\frac{d \widetilde{x}}{d t}(t) & =A \widetilde{x}(t)+B \widetilde{u}(a, t)-L C \widetilde{x}(t), \\
\widetilde{y}(t) & =C \widetilde{x}(t),
\end{aligned}
$$

for $t \in(0, T]$, with initial conditions $\widetilde{x}_{0}=x_{0}-\widehat{x}_{0}$, and

$$
\partial_{t} \widetilde{u}(r, t)=\frac{\epsilon}{r^{m-1}} \partial_{r}\left(r^{m-1} \partial_{r} \widetilde{u}(r, t)\right)-l_{n+1}(r) C \widetilde{x}(t),
$$

for $r \in(a, b), t \in(0, T]$, and boundary conditions

$$
\begin{aligned}
& \partial_{r} \widetilde{u}(a, t)=\beta \widetilde{u}(a, t)+D \widetilde{x}(t)-l_{n+2} C \widetilde{x}(t), \\
& \partial_{r} \widetilde{u}(b, t)=-l_{n+3} C \widetilde{x}(t),
\end{aligned}
$$

with initial conditions $\widetilde{u}_{0}=u_{0}-\widehat{u}_{0}$. Exponential convergence of the estimate $\widehat{x}, \widehat{u}$ to the state $x, u$ is equivalent to the exponential stability of zero solution of the estimation error system. The main result, in Theorem 1, provides a way to compute observer gains $L, l_{n+1}, l_{n+2}$, and $l_{n+3}$, to guarantee exponential stability of the estimation error system. Before the statement of the main result, an additional observability condition is required.

Assumption 1: The finite dimensional subsystem (1), (2) is observable, that is, $\operatorname{rank}(\mathcal{O})=n$, with $\mathcal{O}=$ $\left[\begin{array}{llll}C & C A & \cdots & C A^{n-1}\end{array}\right]^{T}$.

Assumption 1 guarantees the existence of a linear and invertible transformation $T_{\mathcal{O}}: \mathbb{R}^{n} \mapsto \mathbb{R}^{n}$ that maps the system (1)-(2) to the observer canonical form, [14]. The transformation $T_{\mathcal{O}}$ is an invertible matrix, satisfying

$$
T_{\mathcal{O}} A_{\mathcal{O}}=A T_{\mathcal{O}}, \quad T_{\mathcal{O}} B_{\mathcal{O}}=B, \quad C_{\mathcal{O}}=C T_{\mathcal{O}},
$$

where $A_{\mathcal{O}}, B_{\mathcal{O}}, C_{\mathcal{O}}$ are in observer canonical form, that is

$$
\begin{aligned}
A_{\mathcal{O}} & =\left[\begin{array}{ccccc}
a_{1} & 1 & 0 & \cdots & 0 \\
a_{2} & 0 & 1 & \cdots & 0 \\
\vdots & \vdots & \vdots & \ddots & \vdots \\
a_{n-1} & 0 & 0 & \cdots & 1 \\
a_{n} & 0 & 0 & \cdots & 0
\end{array}\right], B_{\mathcal{O}}=\left[\begin{array}{c}
b_{1} \\
b_{2} \\
\vdots \\
b_{n}
\end{array}\right], \\
C_{\mathcal{O}} & =\left[\begin{array}{llll}
1 & 0 & \cdots & 0
\end{array}\right]
\end{aligned}
$$

Assumption 2 (Observability of the Coupled System): None of the eigenvalues $\lambda_{k} \in \mathbb{R}, k \in \mathbb{N}$ of the radial
Laplacian operator with Neumann boundary conditions, that is

$$
\left\{\begin{array}{l}
\epsilon \frac{d}{d r}\left(r^{m-1} \frac{d}{d r} \phi(r)\right)=-\lambda_{k} r^{m-1} \phi(r), \\
\phi_{n}^{\prime}(a)=0 \\
\phi_{n}^{\prime}(b)=0
\end{array}\right.
$$

are, simultaneously, solutions to the polynomial equation $D\left(\lambda_{k}\right)=0$, with

$$
D(\xi)=b_{n}+b_{n-1} \xi+b_{n-2} \xi^{2}+\cdots+b_{2} \xi^{n-2}+b_{1} \xi^{n-1},
$$

and at least one $b_{i}, i \in\{1,2, \cdots, n\}$ is different from zero.

\section{MAIN RESULT}

Before the main result, we describe briefly the methodology followed to define obsever gains that guarantee the stability of the estimation error system.

\section{A. Methodology}

Following the backstepping method for PDEs [1], we seek a pair of transformations $T_{\mathcal{O}}: \mathbb{R}^{n} \rightarrow \mathbb{R}^{n}$ and $T_{u}: \mathcal{L}^{2}(0,1) \times$ $\mathbb{R}^{n} \rightarrow \mathcal{L}^{2}(0,1)$, that map the states $\widetilde{x}, \widetilde{u}$ satisfying (13)-(14), (15)-(17), to states $\widetilde{z}, w$ satisfying the target system

$$
\frac{d \widetilde{z}}{d t}(t)=F \widetilde{z}(t)+B_{\mathcal{O}} w(a, t),
$$

with initial condition $\widetilde{z}_{0}=T_{\mathcal{O}}^{-1} \widetilde{x}_{0}$ and $F \in \mathbb{R}^{n \times n}$ is in companion form, that is

$$
F=\left[\begin{array}{ccccc}
f_{1} & 1 & 0 & \cdots & 0 \\
f_{2} & 0 & 1 & \cdots & 0 \\
\vdots & \vdots & \vdots & \ddots & \vdots \\
f_{n-1} & 0 & 0 & \cdots & 1 \\
f_{n} & 0 & 0 & \cdots & 0
\end{array}\right]
$$

and

$$
\partial_{t} w(r, t)=\frac{\epsilon}{r^{m-1}} \partial_{r}\left(r^{m-1} \partial_{r} w(r, t)\right)-\sigma w(r, t),
$$

for $r \in(a, b), t \in(0, T]$, and boundary conditions

$$
\begin{aligned}
& \partial_{r} w(a, t)=\left(\beta-\frac{\sigma}{2 \epsilon}(b-a)\right) w(a, t), \\
& \partial_{r} w(b, t)=0
\end{aligned}
$$

with initial conditions $w_{0} \in C(a, b)$, satisfying $\widetilde{u}_{0}=$ $T_{w}\left(w_{0}, z_{0}\right)$. The finite-dimensional transformation $T_{\mathcal{O}}$ is defined by (18), (19), while $T_{u}$ is the sum of a secondkind Volterra integral transformation acting on $w$ and a linear spatially-varying transformation acting $z$, that is

$$
\begin{aligned}
\widetilde{x}(t)= & T_{\mathcal{O}} \widetilde{z}(t), \\
\widetilde{u}(r, t)= & w(r, t)-\int_{a}^{r} K(r, s) w(s, t) d s \\
& +(\gamma(r)-\gamma(a)) \widetilde{z}(t),
\end{aligned}
$$

Substitution of (28), (29) in the error (13)-(17) and target systems results in a hyperbolic equation and boundary condition for the kernel $K$ and a differential-algebraic system 
of equation and boundary condition for $\gamma$. Thus, existence of a transformation $T_{u}$ in the form (29) is guaranteed by the existence of a solution to the hyperbolic PDE and DAE systems, which is proven addressed in Lemma 2 and Lemma 3. Invertibility is given by invertibility of $T_{O}$, the triangular structure of the pair (29)-(30) and the fact that the part of the operator $T_{u}$ acting on $w$ is a second-king Volterra integral. To ensure stability of the target system, the eigenvalues of $F$ are selected with negative real part and $\sigma$ is chosen positive, satisfying

$$
\sigma \leq \frac{2 \epsilon \beta}{b-a}
$$

Once the eigenvalues of $F$ and the value of $\sigma$ are chosen, there is a unique value for the observer gains $L, l_{n}$ permitted for consistency of the transformations. These are the observer gains that guarantee the convergence of the estimate to the unknown system state $x, u$, and are presented next in Theorem 1.

\section{B. Main Result}

Theorem 1: Let the premises in Asumption 1 and 2 hold. Consider the estimation error system in (13), (14), (15)-(17), and a similarity transformation $T_{\mathcal{O}} \in \mathbb{R}^{n \times n}$ that maps (1), (2) to observer canonical form (18), (19). Let the ODE observer gain $L \in \mathbb{R}^{n \times 1}$ be chosen such that the eigenvalues $\mu_{i}, i \in$ $\{1, \ldots, n\}$ of the companion (24) matrix $F \in \mathbb{R}^{n \times n}$, defined as

$$
\begin{aligned}
F & =A_{\mathcal{O}}-L_{\mathcal{O}} C_{\mathcal{O}}, \\
L & =T_{\mathcal{O}} L_{\mathcal{O}}
\end{aligned}
$$

have negative real part, and the PDE observer gains $l_{n+1} \in$ $\mathcal{L}^{2}(a, b), l_{n+2} \in \mathbb{R}$, and $l_{n+3} \in \mathbb{R}$ computed from

$$
\begin{aligned}
l_{n+1}(r)= & \epsilon \gamma_{1}^{\prime \prime}(r)+\epsilon \frac{n-1}{r} \gamma_{1}^{\prime}(r) \\
& -\sum_{i=1}^{n}\left(\gamma_{i}(r)-\gamma_{i}(a)\right) f_{i},
\end{aligned}
$$

for $r \in(a, b)$, and

$$
\begin{aligned}
& l_{n+2}=\gamma_{1}^{\prime}(a)-d_{1}, \\
& l_{n+3}=\gamma_{1}^{\prime}(b),
\end{aligned}
$$

where $\gamma_{i}(r), i \in\{1, \ldots, n\}$, is the solution of a differentialalgebraic system of equations

$$
\begin{aligned}
& \epsilon \partial_{s} K(r, a)+\epsilon\left(\beta-\frac{\sigma}{2 \epsilon}(b-a)-\frac{m-1}{a}\right) K(r, a) \\
& +\sum_{i=1}^{n}\left(\gamma_{i}(r)-\gamma_{i}(a)\right) b_{i}=0,
\end{aligned}
$$

for $r \in(a, b)$, and

$$
\begin{aligned}
\frac{\epsilon}{r^{m-1}} \frac{d}{d r}\left(r^{m-1} \frac{d}{d r} \gamma_{2}(r)\right) & =\gamma_{1}(r), \\
\frac{\epsilon}{r^{m-1}} \frac{d}{d r}\left(r^{m-1} \frac{d}{d r} \gamma_{3}(r)\right) & =\gamma_{2}(r), \\
\vdots & \vdots \\
\frac{\epsilon}{r^{m-1}} \frac{d}{d r}\left(r^{m-1} \frac{d}{d r} \gamma_{n}(r)\right) & =\gamma_{n-1}(r),
\end{aligned}
$$

for $r \in(a, b)$, with boundary conditions

$$
\begin{aligned}
& \gamma_{i}^{\prime}(a)=d_{i}, \\
& \gamma_{i}^{\prime}(b)=0,
\end{aligned}
$$

for all $i \in\{2, \ldots, n\}$, where $b_{i}$ are the coefficients of $B_{\mathcal{O}}$ and $d_{i}$ are the coefficients of $D_{\mathcal{O}}=D T_{\mathcal{O}}$. In equation (37), $K \in \mathcal{L}^{2}(\mathcal{T})$ is the solution to a second-order hyperbolic equation

$$
\left\{\begin{array}{l}
\frac{1}{r^{m-1}} \partial_{r}\left(r^{m-1} \partial_{r} K(r, s)\right) \\
-\partial_{s}\left(s^{m-1} \partial_{s}\left(\frac{K(r, s)}{s^{m-1}}\right)\right)=-\frac{\sigma}{\epsilon} K(r, s) \\
\text { with boundary conditions } \\
K(r, r)=\frac{\sigma}{2 \epsilon}(r-b) \\
\partial_{r} K(b, s)=0
\end{array}\right.
$$

with $\sigma$ positive, chosen as

$$
\begin{aligned}
& \underline{\mu} \leq \sigma \leq \frac{2 \epsilon \beta}{b-a}, \\
& \underline{\mu}=\min _{i \in\{1,2, \ldots, n\}}\left\{\left|\mu_{i}\right|\right\} .
\end{aligned}
$$

This choice of observer gains guarantees that estimation error system is exponentially stable, that is

$$
\begin{aligned}
\|\widetilde{x}(t)\|_{2} & \leq \kappa_{1} \exp (-\underline{\mu t})\left(\left\|\widetilde{x}_{0}\right\|_{2}+\left\|\widetilde{u}_{0}\right\|_{\mathcal{H}^{1}}\right), \\
\|\widetilde{u}(\cdot, t)\|_{\mathcal{L}^{2}} & \leq \kappa_{2} \exp (-\underline{\mu} t)\left(\left\|\widetilde{x}_{0}\right\|_{2}+\left\|\widetilde{u}_{0}\right\|_{\mathcal{H}^{1}}\right) .
\end{aligned}
$$

for some positive $\kappa_{1}, \kappa_{2}$.

The well-posedness of the DAE and the hyperbolic PDE are studied in next two lemmas, the proof of Theorem 1 is provided afterwards.

Lemma 2: There is a unique $\mathcal{L}^{2}(\mathcal{T})$ solution to the hyperbolic equation

$$
\left\{\begin{array}{l}
\frac{1}{r^{m-1}} \partial_{r}\left(r^{m-1} \partial_{r} K(r, s)\right) \\
-\partial_{s}\left(s^{m-1} \partial_{s}\left(\frac{K(r, s)}{s^{m-1}}\right)\right)=-\frac{\sigma}{\epsilon} K(r, s), \\
\text { with boundary conditions } \\
K(r, r)=\frac{\sigma}{2 \epsilon}(r-b) \\
\partial_{r} K(b, s)=0
\end{array}\right.
$$

Proof: A solution to hyperbolic system can be found following the procedure described in [16], for kernel equations required for stabilization of diffusion equations in spherical domain, or the procedure described in [17], for kernel equations required for stabilization of diffusion equations with spatially-variable diffusion coefficients. 
Lemma 3: The system of differential algebraic equations (DAE), constituted by $n-1$ differential equations

$$
\begin{aligned}
\frac{\epsilon}{r^{m-1}} \frac{d}{d r}\left(r^{m-1} \frac{d}{d r} \gamma_{2}(r)\right) & =\gamma_{1}(r)-\gamma_{1}(a) \\
\frac{\epsilon}{r^{m-1}} \frac{d}{d r}\left(r^{m-1} \frac{d}{d r} \gamma_{3}(r)\right) & =\gamma_{2}(r)-\gamma_{2}(a) \\
\vdots & \vdots \\
\frac{\epsilon}{r^{m-1}} \frac{d}{d r}\left(r^{m-1} \frac{d}{d r} \gamma_{n}(r)\right) & =\gamma_{n-1}(r)-\gamma_{n-1}(a),
\end{aligned}
$$

with boundary conditions

$$
\begin{aligned}
& \gamma_{i}^{\prime}(a)=d_{i}, \\
& \gamma_{i}^{\prime}(b)=0,
\end{aligned}
$$

for all $i \in\{2, \ldots, n\}$, and by the algebraic equation

$$
\begin{aligned}
& \epsilon \partial_{s} K(r, a)+\epsilon\left(\beta-\frac{\sigma}{2 \epsilon}(b-a)-\frac{m-1}{a}\right) K(r, a) \\
& +\sum_{i=1}^{n}\left(\gamma_{i}(r)-\gamma_{i}(a)\right) b_{i}=0,
\end{aligned}
$$

has a unique solution $\gamma_{i}(r) \in \mathcal{L}^{2}(0,1), i \in\{1, \ldots, n\}$.

Proof: Define

$$
\bar{\gamma}_{i}(r)=\gamma_{i}(r)-\gamma_{i}(a)
$$

for $i \in\{1,2, \ldots, n\}$. By substitution

$$
\begin{aligned}
\frac{\epsilon}{r^{m-1}} \frac{d}{d r}\left(r^{m-1} \frac{d}{d r} \bar{\gamma}_{2}(r)\right) & =\bar{\gamma}_{1}(r), \\
\frac{\epsilon}{r^{m-1}} \frac{d}{d r}\left(r^{m-1} \frac{d}{d r} \bar{\gamma}_{3}(r)\right) & =\bar{\gamma}_{2}(r), \\
\vdots & \vdots \\
\frac{\epsilon}{r^{m-1}} \frac{d}{d r}\left(r^{m-1} \frac{d}{d r} \bar{\gamma}_{n}(r)\right)= & \bar{\gamma}_{n-1}(r),
\end{aligned}
$$

with boundary conditions

$$
\begin{aligned}
& \bar{\gamma}_{i}^{\prime}(a)=d_{i}, \\
& \bar{\gamma}_{i}^{\prime}(b)=0,
\end{aligned}
$$

for all $i \in\{2, \ldots, n\}$, and by the algebraic equation

$$
\begin{aligned}
& \epsilon \partial_{s} K(r, a)-\epsilon\left(\beta-\frac{\sigma}{2 \epsilon}(b-a)-\frac{m-1}{a}\right) K(r, a) \\
& +\sum_{i=1}^{n} \bar{\gamma}_{i}(r) b_{i}=0 .
\end{aligned}
$$

Consider the regular Sturm-Liouville [15] problem

$$
\left\{\begin{array}{l}
\epsilon \frac{d}{d r}\left(r^{m-1} \frac{d}{d r} \phi_{k}(r)\right)=-\lambda_{k} \phi_{k}(r) r^{m-1}, \\
\text { for } r \in(a, b), \text { with boundary conditions } \\
\phi_{k}^{\prime}(a)=0 \\
\phi_{k}^{\prime}(b)=0
\end{array}\right.
$$

for $r \in(a, b)$, with $k \in \mathbb{N}$. The solution to (56) is available as an analytic expression [18],

$$
\phi_{k}(r)=c_{k}\left(r^{-v} J_{v}\left(\mu_{k} r\right)-r^{-v} \frac{J_{v+1}\left(\mu_{k} a\right)}{Y_{v+1}\left(\mu_{k} a\right)} Y_{v}\left(\mu_{k} r\right)\right)
$$

with $v=\frac{m}{2}-1$. The values $\mu_{k} \in \mathbb{R}, k \in \mathbb{N}$ are the solutions to the equation

$$
P_{v+1}\left(\mu_{k} a, \mu_{k} b\right)=0,
$$

where $P_{v}$ is the difference of cross-products of first and second-kind Bessel functions [19],

$$
P_{v}(x, y)=J_{v}(x) Y_{v}(y)-J_{v}(y) Y_{v}(x)
$$

The eigenvalues $\lambda_{k}, k \in \mathbb{N}$ of the Sturm-Liouville problem (56) are

$$
\lambda_{k}=\epsilon \mu_{k}^{2}
$$

Coefficients $c_{k}, k \in \mathbb{N}$ are chosen for normalization, that is

$$
c_{k}=\frac{1}{\left\|r^{-v} J_{v}\left(\mu_{k} r\right)-r^{-v} \frac{J_{v+1}\left(\mu_{k} a\right)}{Y_{v+1}\left(\mu_{k} a\right)} Y_{v}\left(\mu_{k} r\right)\right\|_{\mathcal{L}_{m}^{2}}} \text {. }
$$

The set of functions $\phi_{k}, k \in \mathbb{N}$ form a basis in the Hilbert space $\mathcal{L}_{m}^{2}=\mathcal{L}^{2}\left([a, b], r^{m-1} d r\right)$. Consider a series representation of the functions $\bar{\gamma}_{i}, i \in\{1,2, \cdots, n\}$ in terms of the basis $\phi_{k} \in \mathcal{C}^{\infty}(a, b)$, that is

$$
\bar{\gamma}_{i}(r)=\sum_{k=0}^{\infty} p_{i, k} \phi_{k}(r)
$$

Consider also a series representation for the functions

$$
\begin{gathered}
\frac{\epsilon}{r^{m-1}} \frac{d}{d r}\left(r^{m-1} \frac{d}{d r} \bar{\gamma}_{i}(r)\right), i \in\{2,3, \cdots, n\}, \text { that is } \\
\frac{\epsilon}{r^{m-1}} \frac{d}{d r}\left(r^{m-1} \frac{d}{d r} \bar{\gamma}_{i}(r)\right)=\sum_{k=0}^{\infty} q_{i, k} \phi_{k}(r) .
\end{gathered}
$$

From orthogonality of the basis functions, for all $i \in$ $\{2,3, \cdots, n\}$, and for all $k \in \mathbb{N}$

$$
\begin{aligned}
& \int_{a}^{b}\left(\frac{\epsilon}{r^{m-1}} \frac{d}{d r}\left(r^{m-1} \frac{d}{d r} \bar{\gamma}_{i}(t)\right)-\bar{\gamma}_{i-1}(r)\right) \phi_{k}(r) r^{m-1} d r \\
& =q_{i, k}-p_{i-1, k} .
\end{aligned}
$$

The differential equations (52) are satisfied if and only if the left hand of equation (64) is zero for all $i \in\{2,3, \cdots, n\}$ and for all $k \in \mathbb{N}$, therefore

$$
q_{i, k}=p_{i-1, k}
$$

Similarly, for all $k \in \mathbb{N}$

$$
\begin{gathered}
\int_{a}^{b}\left(\epsilon \partial_{s} K(r, a)+\epsilon\left(\beta-\frac{\sigma}{2 \epsilon}(b-a)-\frac{m-1}{a}\right) K(r, a)\right. \\
\left.\quad+\sum_{i=1}^{n} \bar{\gamma}_{i}(r) b_{i}\right) \times \phi_{k}(r) r^{m-1} d r=\sum_{i=1}^{n} p_{i, k}-z_{k}
\end{gathered}
$$


with

$$
\begin{aligned}
z_{k}=-\int_{a}^{b} & \left(\epsilon \partial_{s} K(r, a)\right. \\
& \left.+\epsilon\left(\beta-\frac{\sigma}{2 \epsilon}(b-a)-\frac{m-1}{a}\right) K(r, a)\right) \times \\
& \phi_{k}(r) r^{m-1} d r .
\end{aligned}
$$

The algebraic equation (55) is satisfied if and only if the lefthand side of equation (66) is zero for all $k \in \mathbb{N}$, therefore

$$
\sum_{i=1}^{n} p_{i, k}=-z_{k}
$$

Further, from (63), orthogonality of the basis functions $\phi_{k}, k \in \mathbb{N}$, and using integration by parts

$$
\begin{aligned}
q_{i, k}= & \int_{a}^{b} \frac{\epsilon}{r^{m-1}} \frac{d}{d r}\left(r^{m-1} \frac{d}{d r} \bar{\gamma}_{i}(r)\right) \phi_{k}(r) r^{m-1} d r \\
= & \epsilon \int_{a}^{b} \frac{d}{d r}\left(r^{m-1} \frac{d}{d r} \bar{\gamma}_{i}(r)\right) \phi_{k}(r) d r \\
= & \left.\epsilon r^{m-1} \frac{d}{d r} \bar{\gamma}_{i}(r) \phi_{k}(r)\right|_{a} ^{b} \\
& -\epsilon \int_{a}^{b} r^{m-1} \frac{d}{d r} \bar{\gamma}_{i}(r) \frac{d}{d r} \phi_{k}(r) d r
\end{aligned}
$$

using boundary conditions for $\bar{\gamma}_{i}(r)$

$$
\begin{aligned}
q_{i, k}= & -\epsilon a^{m-1} d_{i} \phi_{k}(a)-\left.\epsilon r^{m-1} \bar{\gamma}_{i}(r) \frac{d}{d r} \phi_{k}(r)\right|_{a} ^{b} \\
& +\epsilon \int_{a}^{b} \bar{\gamma}_{i}(r) \frac{d}{d r}\left(r^{m-1} \frac{d}{d r} \phi_{k}(r)\right) d r
\end{aligned}
$$

the equation and boundary conditions for $\phi_{k}(r)$ result in

$$
q_{i, k}=-\epsilon a^{m-1} d_{i} \phi_{k}(a)-\lambda_{k} \int_{a}^{b} \bar{\gamma}_{i}(r) \phi_{k}(r) r^{m-1} d r
$$

from definition of $p_{i, k}$

$$
q_{i, k}=-\epsilon a^{m-1} d_{i} \phi_{k}(a)-\lambda_{k} p_{i, k},
$$

substitution of (75) in (65) implies

$$
\lambda_{k} p_{m}+p_{i-1, k}=-\epsilon a^{m-1} d_{i} \phi_{k}(a) .
$$

For each $k \in \mathbb{N}$, the $n-1$ algebraic equations (76) together with the equation (68) result in a $n$-dimensional algebraic system that one needs to solve in order to compute $\left\{q_{1, k}, q_{2, k}, \cdots, q_{n, k}\right\}$. In other words, the coefficients in the series (62) should satisfy an infinite sequence of $n$ dimensional linear systems

$$
\left[\begin{array}{ccccc}
\lambda_{k} & 1 & \cdots & 0 & 0 \\
0 & \lambda_{k} & \cdots & 0 & 0 \\
\vdots & \vdots & \ddots & \vdots & \vdots \\
0 & 0 & \cdots & \lambda_{k} & 1 \\
b_{n} & b_{n-1} & \cdots & b_{2} & b_{1}
\end{array}\right]\left[\begin{array}{l}
p_{n, k} \\
p_{n-1, k} \\
\vdots \\
p_{2, k} \\
p_{1, k}
\end{array}\right]=\left[\begin{array}{l}
\beta_{k} d_{n} \\
\beta_{k} d_{n-1} \\
\vdots \\
\beta_{k} d_{2} \\
z_{k}
\end{array}\right]
$$

where

$$
\begin{aligned}
\beta_{k} & =-\epsilon a^{m-1} \phi_{k}(a) \\
z_{k}= & -\int_{a}^{b}\left(\epsilon \partial_{s} K(r, a)\right. \\
& \left.+\epsilon\left(\beta-\frac{\sigma}{2 \epsilon}(b-a)-\frac{m-1}{a}\right) K(r, a)\right) \\
& \times \phi_{k}(r) r^{m-1} d r .
\end{aligned}
$$

Note that the generalized Fourier coefficients define a function uniquely in the space of definition, that is $\mathcal{L}^{2}\left([a, b], r^{m-1} d r\right)$, for that reason, a unique solution of the algebraic system in the is a necessary and sufficient condition for the existence of a solution. Define

$$
\begin{gathered}
D(\xi)=\operatorname{det}\left(\left[\begin{array}{cccccc}
\xi & 1 & 0 & \cdots & 0 & 0 \\
0 & \xi & 1 & \cdots & 0 & 0 \\
0 & 0 & \xi & \cdots & 0 & 0 \\
\vdots & \vdots & \vdots & \ddots & \vdots & \vdots \\
0 & 0 & 0 & \cdots & \xi & 1 \\
b_{n} & b_{n-1} & b_{n-2} & \cdots & b_{2} & b_{1}
\end{array}\right]\right) \\
=b_{n}+b_{n-1} \xi+b_{n-2} \xi^{2}+\cdots+b_{2} \xi^{n-2}+b_{1} \xi^{n-1} .
\end{gathered}
$$

The sequence of linear systems (77) has a unique solution $q_{i, k}$, with $i \in\{1,2, \ldots, n\}, k \in \mathbb{N}$, if and only if

$$
D\left(\lambda_{k}\right) \neq 0,
$$

for all $k \in \mathbb{N}$. This condition is related to the observability of the coupled system, since it defines a cancellation between the spectral values of the PDE and zeros of the ODE. This condition appears in Assumption 2.

\section{Proof of Theorem 1}

Proof: The stability is then verified with Lyapunov-like functions

$$
\begin{aligned}
& V_{1}(t)=\frac{1}{2} \int_{a}^{b} w^{2}(r, t) r^{m-1} d r \\
& V_{2}(t)=\frac{\epsilon \theta}{2} w(a, t)^{2} a^{m-1}+\frac{1}{2} \int_{a}^{b} w_{r}^{2}(r, t) r^{m-1} d r
\end{aligned}
$$

with

$$
\theta=\beta-\frac{\sigma}{2 \epsilon}(b-a),
$$

which satisfies $\theta>0$, according to the condition (30). The time derivatives of $V_{1}(t)$ and $V_{2}(t)$ along the trajectories of the $w$-system are

$$
\begin{aligned}
\frac{d V_{1}}{d t}(t)= & \left.\epsilon w(r, t) \partial_{r} w(r, t) r^{m-1}\right|_{a} ^{b} \\
& -\epsilon \int_{a}^{b}\left(\partial_{r} w(r, t)\right)^{2} r^{m-1} d r-\sigma \int_{a}^{b} w(r, t)^{2} r^{m-1} d r
\end{aligned}
$$

then,

$$
\frac{d V_{1}}{d t}(t) \leq-2 V_{1}(t)
$$


The time derivate of $V_{2}(t)$ is

$$
\begin{aligned}
\frac{d V_{2}}{d t}(t) \leq & -\epsilon \theta \sigma w(a, t)^{2} a^{m-1} \\
& -\epsilon \int_{a}^{b}\left(\frac{1}{r^{m-1}} \partial_{r}\left(r^{m-1} \partial_{r} w(r, t)\right)\right)^{2} r^{m-1} d r \\
& -\sigma \int_{a}^{b}\left(\partial_{r} w(r, t)\right)^{2} r^{m-1} d r \\
\leq & -\epsilon \theta \sigma w(a, t)^{2} a^{m-1}-\sigma \int_{a}^{b}\left(\partial_{r} w(r, t)\right)^{2} r^{m-1} d r
\end{aligned}
$$

Therefore,

$$
\frac{d V_{2}}{d t}(t) \leq-2 \sigma V_{2}(t)
$$

From comparison principle

$$
\begin{aligned}
& V_{1}(t) \leq \exp [-2 \sigma t] V_{1}(0), \\
& V_{2}(t) \leq \exp [-2 \sigma t] V_{2}(0),
\end{aligned}
$$

and consequently,

$$
\begin{aligned}
\|w(\cdot, t)\|_{\mathcal{L}^{2}} & \leq \exp [-\sigma t]\left\|w_{0}\right\|_{\mathcal{L}^{2}}, \\
|w(a, t)| & \leq \exp [-\sigma t]\left(\left|w_{0}(a)\right|+k_{1}\left\|\partial_{r} w_{0}\right\|_{\mathcal{L}^{2}}\right),
\end{aligned}
$$

with

$$
k_{1}=\frac{a^{-\frac{(m-1)}{2}}}{\sqrt{\epsilon \theta}} .
$$

From Lemma 4 in the Appendix,

$$
|w(a, t)| \leq k_{2} \exp [-\sigma t]\left\|w_{0}\right\|_{\mathcal{H}^{1}} .
$$

with $k_{2}=\max \left\{1, k_{1}\right\}$. The observer gain $L=$ $\left[l_{1}, l_{2}, \ldots, l_{n}\right]^{T}$ is chosen via pole placement. That is, given a set of $n$ complex-valued numbers $\left\{\mu_{1}, \mu_{2}, \ldots, \mu_{n}\right\}$ with negative real parts, it is always possible to find $L$ such that the eigenvalues of $F=A-L C$, are exactly $\left\{\mu_{1}, \mu_{2}, \ldots, \mu_{n}\right\}$. From the variation of constants formula

$$
\widetilde{z}(t)=\exp [F t] \widetilde{z}_{0} \int_{0}^{t} \exp [F(t-\tau)] B w(a, \tau) d \tau .
$$

Then, there exits $k_{3}>0$, such that the norm of the state $\widetilde{x}(t)$ is bounded as follows

$$
\begin{aligned}
\|\widetilde{z}(t)\|_{2} \leq & k_{3} \exp [-\underline{\mu} t]\left|\widetilde{z}_{0}\right|_{2} \\
& +k_{3}\|B\|_{2} \int_{0}^{t} \exp [-\underline{\mu}(t-\tau)]|w(a, \tau)| d \tau,
\end{aligned}
$$

with $\underline{\mu}=\min _{i \in\{1,2, \ldots, n\}}\left\{\left|\mu_{i}\right|\right\}$. From (30), it follows that $\sigma>\underline{\mu}>0$, and therefore

$$
\|\widetilde{z}(t)\|_{2} \leq k_{3} \exp [-\underline{\mu} t] \widetilde{z}_{0}+\frac{k_{2} k_{3}}{\sigma-\underline{\mu}} \exp [-\underline{\mu t}]\|B\|_{2}\left\|w_{0}\right\|_{H_{1}} .
$$

The inequalities imply that the zero solution of the target system is stable, with exponential bounds

$$
\begin{aligned}
\|w(\cdot, t)\|_{\mathcal{L}^{2}} & \leq \exp [-\sigma t]\left\|w_{0}\right\|_{\mathcal{L}^{2}}, \\
|\widetilde{z}(t)|_{2} & \leq k_{3} \exp [-\underline{\mu t}]\left|\widetilde{z}_{0}\right|_{2}+k_{4} \exp [-\underline{\mu t}]\left\|w_{0}\right\|_{H_{1}} .
\end{aligned}
$$

with

$$
k_{4}=\frac{k_{2} k_{3}}{\sigma-\underline{\mu}}\|B\|_{2} .
$$

Since the pair of transformation $T_{\mathcal{O}}$ and $T_{u}$ defined in (18), (28) and (29) are invertible and bounded, there exists positive $\kappa_{1}, \kappa_{2}$, such that

$$
\begin{aligned}
\|\widetilde{x}(t)\|_{2} \leq \kappa_{1} \exp (-\underline{\mu t})\left(\left\|\widetilde{x}_{0}\right\|_{2}+\left\|\widetilde{u}_{0}\right\|_{\mathcal{H}^{1}}\right), \\
\|\widetilde{u}(\cdot, t)\|_{\mathcal{L}^{2}} \leq \kappa_{2} \exp (-\underline{\mu} t)\left(\left\|\widetilde{x}_{0}\right\|_{2}+\left\|\widetilde{u}_{0}\right\|_{\mathcal{H}^{1}}\right) .
\end{aligned}
$$

\section{WellBore AND RESERVOIR MOdel}

The process of oil well drilling consists in creating a borehole several kilometres into the ground. Throughout the process, a drilling fluid (typically oil- or water-based mud) is circulated to lubricate and cool the drilling tools, evacuate mud cuttings and pressurize the well. The mud is pumped through the drillstring, flows through the drillbit and travels up the annular region, as schematically depicted on Figure IV-A. When the pressure at the bottom of the well is lower than the pressure of the reservoir surrounding it, an influx of liquid, and potentially gas, will enter the annulus. We apply the observer design of the previous sections to estimate, from surface measurements only, the flow rate of a liquid influx as well as the near-wellbore reservoir pressure profile.

\section{A. Model}

The finite-dimensional part of the following model is an adaptation from [21] to account for the liquid influx while the reservoir PDE is described in [2]. The model derives from first principles and the states $x(t)=\left[p_{c}(t), p_{p}(t), q_{d}(t)\right]^{\prime}$ and $u(r, t), r \in\left[r_{w}, r_{e}\right]$ denote, respectively, the pressure upstream the outlet choke, the pump pressure, the total influx into the annulus and the reservoir pressure profile. The model takes the following form

$$
\begin{aligned}
\frac{d x(t)}{d t} & =A x(t)+B u\left(r_{w}, t\right), \\
y(t) & =C x(t), \\
\partial_{t} u(r, t) & =\frac{\epsilon}{r} \partial_{r}\left(r \partial_{r} u(r, t)\right), \\
\partial_{r} u\left(r_{w}, t\right) & =\beta u\left(r_{w}, t\right)+D x(t), \\
\partial_{r} u\left(r_{e}, t\right) & =0,
\end{aligned}
$$

where

$$
\begin{aligned}
A & =\left[\begin{array}{ccc}
a_{0} & 0 & 0 \\
0 & 0 & a_{1} \\
0 & a_{2} & a_{3}
\end{array}\right], B=\left[\begin{array}{c}
b_{0} \\
0 \\
b_{1}
\end{array}\right], \\
D & =\left[\begin{array}{lll}
d_{0} & 0 & d_{1}
\end{array}\right] .
\end{aligned}
$$

Definition of the matrix elements are given in Table I and the physical parameters are given in Table II. Two measurements are typically available on drilling facilities, namely pump and choke pressure, which yields

$$
C=\left[\begin{array}{lll}
1 & 0 & 0 \\
0 & 1 & 0
\end{array}\right] \text {. }
$$




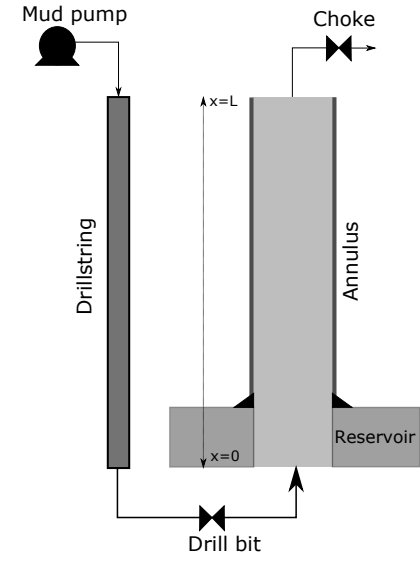

Fig. 1. Schematic of fluid flow-path in a wellbore

TABLE I

DEFINITION OF MATRIX ELEMENTS

\begin{tabular}{|c|c||c|c|}
\hline Element & Definition & Element & Definition \\
\hline$a_{0}$ & $-\frac{\beta_{a}}{V_{a}}\left(\frac{A_{a} D_{a}^{2}}{32 \mu H}+\frac{K_{c} \bar{Z}_{c}}{\sqrt{2 \rho_{0}\left(\bar{p}_{c}-p_{0}\right)}}\right)$ & $a_{1}$ & $-\frac{\beta_{d}}{V_{d}}$ \\
\hline$a_{3}$ & $-\frac{32 \mu H}{M_{d} A_{d} D_{d}^{2}}+\frac{\rho_{0} \bar{q}_{d}}{M_{d} C_{d}^{2} A_{n}^{2}}$ & $a_{2}$ & $\frac{1}{M_{d}}$ \\
\hline$b_{0}$ & $\frac{\beta_{a}}{V_{a}}\left(\frac{A_{a} D_{a}^{2}}{32 \mu H}\right)$ & $b_{1}$ & $-\frac{1}{M_{d}}$ \\
\hline$d_{0}$ & $-\frac{A_{a} D_{a}^{2}}{32 \mu \xi H}$ & $d_{1}$ & $-\frac{1}{\xi}$ \\
\hline
\end{tabular}

These two measurements are required to make the pair $(A, C)$ observable. Thus, the design of Section II has to be slightly adapted to account for multiple outputs. We omit the details here for the sake of brevity, however, they pose no difficulty using a block observer canonical form, since the presence of multiple outputs only adds degrees of freedom to estimate the state of the scalar PDE.

\section{B. Numerical simulations}

A test case, where the opening of the choke valve (see Figure IV-B) is increased suddenly, is considered here to illustrate the results. This leads to reduction of the pressure at the bottom of the wellbore and thus result in an influx from the reservoir. Figure IV-B shows the comparison between the influx from the plant, the influx estimated by the observer and an open-loop estimation. Since the dynamics are stable, one could expect the latter to provide an asymptotic estimate of the influx. This is not the case, however, due to the slow time-scale of the reservoir dynamics. A similar result is seen in estimating the near-wellbore reservoir profile as depicted in Figure IV-B which is a snapshot of the reservoir pressure profile at $60 \mathrm{~s}$.

\section{CONCLUSION}

We have derived an observer for coupled ODE-PDE system with bidirectional coupling. The observer is derived following the backstepping method for PDE, i.e. defining an integral transformation that maps the estimation error
TABLE II

DEFINITION OF WELLBORE AND FLUID PARAMETERS

\begin{tabular}{|c||c||c||c|}
\hline Parameter & Definition & Value & Unit \\
\hline$\beta_{a, d}$ & Bulk modulus & $6.896 \mathrm{e} 8$ & $P a$ \\
\hline$D_{a}$ & Annulus diameter & 0.1809 & $m$ \\
\hline$D_{d}$ & Drillstring diameter & 0.1143 & $m$ \\
\hline$H$ & Depth of the well & 2000 & $m$ \\
\hline$\mu$ & Mud viscosity & $40 \mathrm{e}-2$ & $P a . s$ \\
\hline$K_{c}$ & Choke constant & 0.0029 & {$[-]$} \\
\hline$Z_{c}$ & Steady state choke opening & 0.8 & {$[-]$} \\
\hline $\bar{p}_{c}$ & Steady state choke pressure & $1.675 \mathrm{e} 5$ & $P a$ \\
\hline$C_{d}$ & Bit nozzle constant & 0.8 & {$[-]$} \\
\hline$A_{n}$ & Bit nozzle area & $7.459 \mathrm{e}-4$ & $\mathrm{~m}^{2}$ \\
\hline$p_{o}$ & Reference pressure & $1 \mathrm{e} 5$ & $\mathrm{~Pa}$ \\
\hline$\rho_{o}$ & Reference density & 780 & $\mathrm{~kg} / \mathrm{m}^{3}$ \\
\hline$\kappa$ & Permeability & $5 \mathrm{e}-12$ & $\mathrm{~m}^{2}$ \\
\hline$\phi$ & Porosity & 0.2 & {$[-]$} \\
\hline$c_{t}$ & Total compressibility (reservoir) & $2.32 \mathrm{e}-9$ & $\mathrm{~Pa}^{-1}$ \\
\hline
\end{tabular}

\begin{tabular}{|c|c|}
\hline Parameter & Defintion \\
\hline$A_{a, d}$ & Area \\
\hline$V_{a, d}$ & Volume \\
\hline$r_{w}$ & Radius of annulus \\
\hline$\xi$ & $2 \pi \kappa r_{w} H / \mu$ \\
\hline$M_{d}$ & $\begin{array}{c}\text { Integrated density per cross section, } \\
\int_{0}^{L}\left(\rho(x) / A_{d}(x)\right) d x\end{array}$ \\
\hline$\beta$ & $-d_{0} / \xi$ \\
\hline$\epsilon$ & Diffusivity constant, $\epsilon=\kappa /\left(\mu c_{t} \phi\right)$ \\
\hline Subscripts $a, d$ & annulus, drillstring \\
\hline
\end{tabular}

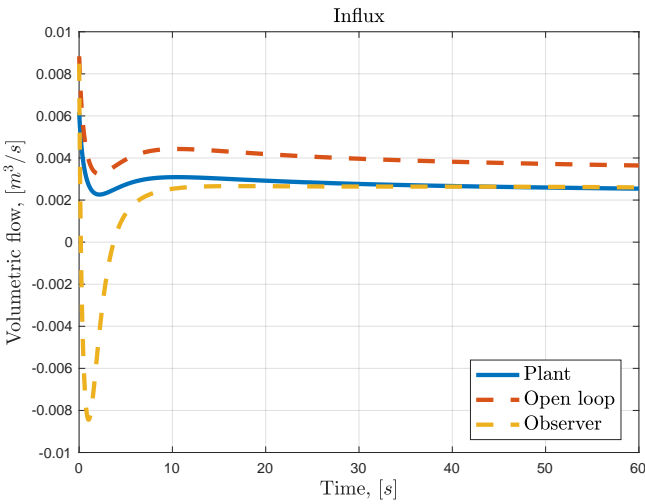

Fig. 2. Observer performance to estimate the influx into the wellbore

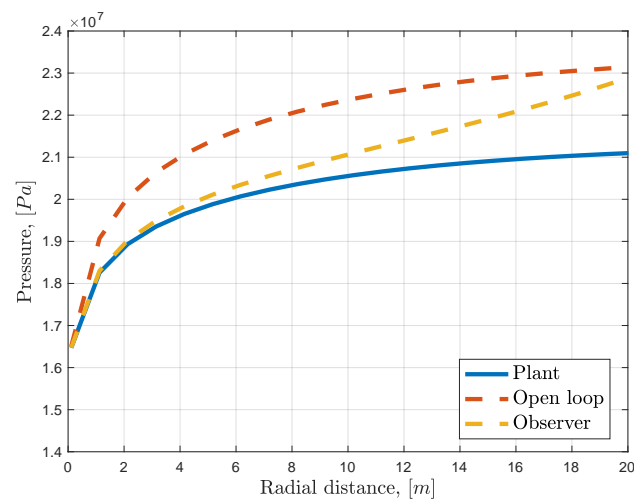

Fig. 3. Observer performance to estimate the near-wellbore pressure profile 
system to a stable target system. Interestingly, the wellposedness conditions for the kernel equations are exactly the conditions of observability of the coupled system. The design is applied to a model of wellbore-reservoir dynamics, used in the managed pressured drilling application. This allows us to estimate the influx from the reservoir by only using the measurements of the finite dimensional states that are typically available at the surface of drilling rigs.

The main shortcoming of the current design is the inability to modify the coefficient of the Robin boundary condition at the PDE-ODE interface. Future works include the modification of the transformation to compensate for this. Besides, the design of spatially-varying source terms in the PDE target system and a proper choice of closed-loop eigenvalues could be used to decrease the overshoot in the transient state estimation dynamics.

\section{APPENDIX}

Lemma 4: For any function $f \in C([a, b])$,

$$
f(a) \leq \kappa_{a}\|f\|_{\mathcal{H}^{1}}
$$

with $\kappa_{a}=\frac{(b-a)^{2}+1}{b-a}$.

Proof: From the fundamental theorem of calculus and triangle inequality

$$
|f(a)| \leq|f(r)|+\int_{a}^{b}\left|\partial_{\xi} f(\xi)\right| d \xi
$$

for any $r \in[a, b]$. Using Cauchy-Swartz inequality

$$
|f(a)| \leq|f(r)|+\sqrt{b-a} \sqrt{\int_{a}^{b}\left|\partial_{r} f(r)\right|^{2} d r}
$$

using Young's inequality

$$
|f(a)|^{2} \leq\left(\frac{\Xi+1}{\Xi}\right)|f(r)|^{2}+2(b-a)(1+\Xi) \int_{a}^{b}\left|\partial_{r} f(r)\right|^{2} d r .
$$

Since the last inequality holds for all $r \in[a, b]$, it follows that

$$
\begin{aligned}
(b-a)|f(a)|^{2} \leq & \frac{\Xi+1}{\Xi} \int_{a}^{b}|f(r)|^{2} d r \\
& +(1+\Xi)(b-a)^{2} \int_{a}^{b}\left|\partial_{r} f(r)\right|^{2} d r
\end{aligned}
$$

for some $\Xi>0$. Diving both side by $b-a$,

$$
\begin{aligned}
|f(a)|^{2} \leq & \frac{\Xi+1}{\Xi} \frac{1}{b-a} \int_{a}^{b}|f(r)|^{2} d r \\
& +(1+\Xi)(b-a) \int_{a}^{b}\left|\partial_{r} f(r)\right|^{2} d r
\end{aligned}
$$

Choosing $\Xi=(b-a)^{-2}$, one obtains

$$
|f(a)| \leq \kappa_{a}\|f\|_{H^{1}}
$$

with $\kappa_{a}=\frac{(b-a)^{2}+1}{b-a}$.

\section{ACKNOWLEDGMENT}

Leobardo Camacho-Solorio acknowledges support from the Chateaubriand Fellowship, UC-MEXUS, and CONACYT.

Naveen Velmurugan and Florent Di Meglio acknowledge support from the HYDRA project, which has received funding from the European Union's Horizon 2020 research and innovation program under grant agreement No 675731.

\section{REFERENCES}

[1] M. Krstic and A. Smyshlyaev, Boundary Control of PDEs, Society of Industrial and Applied Mathematics, 2008.

[2] N. Velmurugan and F. Di Meglio, "Transient Modelling of Influx and Observer Implementation for Estimation While Drilling, " Conference on Decision and Control, 2018.

[3] M. Krstic and A. Smyshlyaev, "Backstepping Boundary Control for First-Order Hyperbolic PDEs and Application to Systems with Actuator and Sensor Delays", Systems \& Control Letters, vol. 57, pp. 750-758, 2008.

[4] M. Krstic, Delay Compensation for Nonlinear, Adaptive, and PDE Systems, Birkhauser, 2009.

[5] M. Krstic, "Compensating a String PDE in the Actuation or Sensing Path of an Unstable ODE," American Control Conference, 2009.

[6] M. Krstic, "Predictor-like Feedback for Actuator and Sensor Dynamics Governed by Diffusion PDEs", American Control Conference, 2009.

[7] G.A. Susto and M. Krstic, "Control of PDE-ODE Cascades with Neumann Interconnections," Journal of the Franklin Institute, vol. 347, pp. 284-314, 2010.

[8] J. Wang, M. Krstic, and Y. Pi, "Control of a $2 \times 2$ coupled linear hyperbolic system sandwiched between 2 ODEs," International Journal of Robust and Nonlinear Control, vol. 28, pp. 3987-4016, 2018.

[9] N. Bekiaris-Liberis and M. Krstic, "Compensating the Distributed Effect of Diffusion and Counter-Convection in Multi-Input and MultiOutput LTI Systems", IEEE Transactions on Automatic Control, vol. 56, pp. 637-643, 2010.

[10] S. Tang and C. Xie, "State and Output Feedback Boundary Control for a Coupled PDE-ODE System, " Systems \& Control Letters, vol. 60, pp. 540-545, 2011.

[11] B. Ren, J.M. Wang and M. Krstic, "Stabilization of an ODESchrdinger Cascade, " Systems \& Control Letters, vol. 62, pp. 503-510, 2013.

[12] A. Hasan, O.M. Aamo, and M. Krstic, "Boundary Observer Design for Hyperbolic PDE-ODE Cascade Systems, " Automatica, vol. 68, pp 75-86, 2016.

[13] F. Di Meglio, F. Bribiesca Argomedo, L. Hu, and M. Krstic, "Stabilization of Coupled Linear Heterodirectional Hyperbolic PDE-ODE systems, " Automatica, vol. 87, pp. 281-289, 2018.

[14] T. Kailath, Linear Systems, Prentice-Hall, Englewood Cliffs, NJ, 1980.

[15] Zettl, Anton, Sturm-Liouville Theory, American Mathematical Society, 2005.

[16] R. Vazquez and M. Krstic, "Boundary control and estimation of reaction-diffusion equations on the sphere under revolution symmetry conditions", International Journal of Control, 2017

[17] J.M. Coron, and H.M. Nguyen, "Null Controllability and Finite Time Stabilization for the Heat Equations with Variable Coefficients in Space in One Dimension via Backstepping Approach", Archive for Rational Mechanics Analysis, vol 225, pp. 993-1023, 2017

[18] L. Camacho-Solorio, S. Moura, M. Krstic, "Robustness of Boundary Observers for Radial Diffusion Equations to Parameter Uncertainty," American Control Conference, 2018.

[19] D. Kirkham, "Graphs and Formulas for Zeros of Cross Product Bessel Functions," Journal of Mathematics and Physics, vol. 36, pp.371-377, 1957.

[20] G. N. Watson, A Treatise on the Theory of Bessel Functions, Cambridge University Press, 1995.

[21] G. O., Kaasa, . N Stamnes O. M. Aamo, and L. S. Imsland, "Simplified hydraulics model used for intelligent estimation of downhole pressure for a managed-pressure-drilling control system", SPE Drilling \& Completion, vol. 27, pp. 127-138, 2012. 